\title{
LETTER \\ Approximated Virtual Source Imaging System for a Pair of Closely Spaced Loudspeakers
}

\author{
Jae-woong JEONG ${ }^{\dagger \text { a) }}$, Nonmember, Young-cheol PARK ${ }^{\dagger \dagger}$, Member, and Dae-hee YOUN ${ }^{\dagger}$, Nonmember
}

\begin{abstract}
SUMMARY This paper presents an approximated virtual source imaging system based on crosstalk cancellation with a pair of closely spaced loudspeakers. Utilizing the frequency-dependent relative importance of sound localization cues, the proposed system provides separate approximations for the low- and high-frequency bands. Experimental results show that the system provides good approximations within $\pm 55^{\circ}$ in the stereo dipole setup with natural sound quality.

key words: sound cues, stereo dipole, virtual source imaging
\end{abstract}

\section{Introduction}

When a virtual source imaging (VSI) system is realized with a pair of closely spaced loudspeakers, the main issue is how to faithfully reproduce virtual sound images particularly at the outside angle spanned by the loudspeakers. Among a number of algorithms to accomplish this task, the crosstalk cancellation (CTC) technique can, theoretically, provide the most accurate localization [1]. However, the traditional CTC systems are often associated with computational issues [2] and timbral distortions of the input signals [3].

To overcome these problems, we try to approximate the CTC filters with the interaural level differences (ILDs) and the interaural time differences (ITDs) parameters. The approximation model in this paper is based on the previous work in [4] where the approximation was conducted for a general stereo loudspeaker setup. In this paper, the method in [4] is applied to the configuration of a pair of closely spaced loudspeakers, which results in a sophisticated model that can nicely approximate the CTC-based VSI system using a few control parameters, while preventing severe timbral distortions of the input signals.

The remaining sections are organized as follows: Sects. 2 and 3 describe the conventional and proposed VSI systems for a pair of closely spaced loudspeakers, respectively. Sections 4 and 5 present performance evaluations via a series of experiments and conclusions, respectively.

\section{Conventional VSI Systems}

Figure 1 shows the geometrical arrangement of loudspeakers for the VSI system where the speaker angle is denoted by $\theta$ and the virtual source angle is denoted by $\phi$. In this

\footnotetext{
Manuscript received April 15, 2014.

${ }^{\dagger}$ The authors are with Yonsei Univ., Seoul, Korea.

${ }^{\dagger \dagger}$ The author is with Yonsei Univ., Wonju, Kangwon, Korea.

a) E-mail: woong@dsp.yonsei.ac.kr

DOI: 10.1587/transinf.2014EDL8073
}
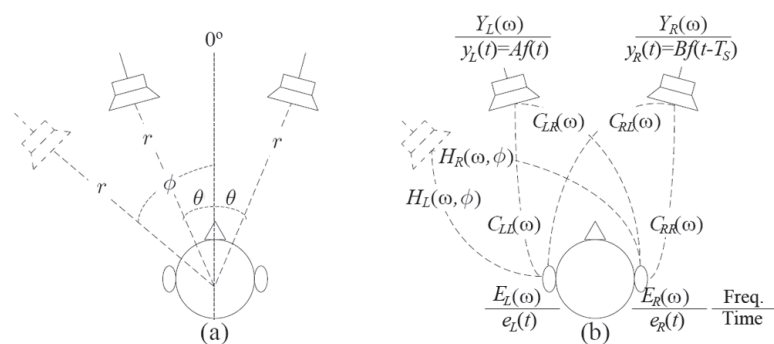

Fig. 1 (a) arrangement of loudspeakers, and (b) definitions of signals.

paper, it is assumed that the loudspeakers are placed very closely, i.e. stereo dipole [1], and thus $\theta$ is almost always smaller than $\phi$. Both loudspeaker signals are denoted by $Y_{L}(\omega)$ and $Y_{R}(\omega)$ in the frequency domain, and $y_{L}(t)$ and $y_{R}(t)$ in the time domain. Likewise, the signals arriving at both ear positions of the listener are denoted by $E_{L}(\omega)$ and $E_{R}(\omega)\left(e_{L}(t)\right.$ and $\left.e_{R}(t)\right)$. The head-related transfer functions (HRTFs) from the loudspeakers to the listener are denoted by $C_{L L}(\omega), C_{L R}(\omega), C_{R L}(\omega)$ and $C_{R R}(\omega)$, respectively. Also, $H_{L}(\omega)$ and $H_{R}(\omega)$ are the HRTFs between the desired virtual source and the listener.

The essential requirement for the VSI is that the summed signals from the loudspeakers at the listener's ear should be identical to the ear response to the virtual source. Then, the loudspeaker signals are obtained as [2]

$$
\left[\begin{array}{l}
Y_{L}(\omega) \\
Y_{R}(\omega)
\end{array}\right]=\frac{1}{\kappa}\left[\begin{array}{cc}
C_{R R}(\omega) & -C_{L R}(\omega) \\
-C_{R L}(\omega) & C_{L L}(\omega)
\end{array}\right]\left[\begin{array}{c}
H_{L}(\omega) \\
H_{R}(\omega)
\end{array}\right],
$$

where $\kappa=C_{L L}(\omega) C_{R R}(\omega)-C_{L R}(\omega) C_{R L}(\omega)$. This procedure is known as the crosstalk cancellation. In theory, since HRTFs are perfectly inverted by using the CTC filters, exact localization is achieved without any timberal distortion. However, when public HRTFs are utilized, undesirable frequency boosting or cutting occurs in the ear signal and which can result in timbral distortions [3]. In addition, due to HRTFs with long time responses, the traditional CTC system have computational issues that need to be taken into consideration [2]. To remedy these problems, several algorithms for modifying or approximating the CTC network have been suggested [4]-[6]. Among these algorithms, Kirkeby et al. [6] proposed a VSI method based on the stereo dipole where the CTC filters were parameterized using ILDs and ITDs of the HRTFs. In this method, high frequency ILD due to the virtual source was assumed to be much larger than unity. However, this assumption holds only for the virtual source located at relatively wide angles. Thus, the corre- 
sponding VSI system might distorts sound localization and quality of the virtual source at relatively narrow angle.

\section{Proposed VSI System}

Recognizing the shortcomings of Kirkeby's method, we try different approximations that are applicable to general cases where no particular assumption is made about the ILD and ITD parameters. Similar to the method in [4], the CTC filters in this paper is approximated in two separate bands split at a frequency of $1.5 \mathrm{kHz}$. The split frequency is determined from the "duplex theory" [7] which states that the ITDs are prominent for sound localization at frequencies lower than about $1.5 \mathrm{kHz}$, whereas the ILDs are important at frequencies higher than $1.5 \mathrm{kHz}$.

From Eq. (1), a ratio between the loudspeaker signals is defined as $K(\omega)=\frac{Y_{L}(\omega)}{Y_{R}(\omega)}$. At low frequencies, this can be approximated as $K(\omega) \approx \frac{T_{a}+\tau}{T_{a}-\tau}$ [4] where $T_{a}$ and $\tau$ represent ITDs due to the loudspeaker and virtual source, respectively. Unlike the case of the general stereo loudspeaker setup in [4] where $\tau<T_{a}$, a virtual source at the outside angle spanned by a pair of closely spaced loudspeakers yields a situation where $\tau>T_{a}$. Thus, $K(\omega)$ becomes a frequencyindependent negative constant:

$$
K\left(\omega_{l}\right)=k_{l} \approx-\left(\frac{\tau+T_{a}}{\tau-T_{a}}\right),
$$

where $\omega_{l} \in[0,2 \pi \cdot 1.5 \mathrm{kHz}]$. The significance of Eq. (2) is that, at low frequencies, the CTC filters are approximated to the well-known amplitude panning [8] but with a changed sign due to that the virtual source position $(\phi)$ is almost always wider than the loudspeaker angle $(\theta)$. This property was referred to as "duality property" in [6], where Kirkeby's method used the same model in Eq. (2) at low frequencies.

In the high-frequency band, the CTC filter is approximated based on the fact that the "envelope" function of the waveform is important for high-frequency localization [9]. The loudspeaker signal ratio $K(\omega)$ can be approximated using a level ratio $k_{h}$ and the time difference $T_{s}$ between the loudspeaker signals, respectively [4]. For the frequency $\omega_{h} /(2 \pi) \in\left[1.5 \mathrm{kHz}, F_{s} / 2\right]$ with a sampling frequency $F_{s}$, the level ratio is obtained as a squared form:

$$
\left|K\left(\omega_{h}\right)\right|^{2}=k_{h}^{2}=\frac{y_{L}^{2}(t)}{y_{R}^{2}(t)} \approx \frac{m_{h}^{2} \Delta_{h}^{2}-1}{m_{h}^{2}-\Delta_{h}^{2}},
$$

where $\Delta_{h}$ denotes the high-frequency ILD resulting from both left and right loudspeakers, and $m_{h}$ denotes the highfrequency ILDs due to each loudspeaker signal. The time difference can be computed as [4]

$$
T_{s} \approx \frac{\left(k_{h}^{4} m_{h}^{2}+m_{h}^{2} m_{h}^{4}+k_{h}^{2}+m_{h}^{2}\right) \tau_{h}-\left(k_{h}^{4} m_{h}^{2}-m_{h}^{2}\right) T_{a}}{k_{h}^{2} m_{h}^{4}-k_{h}^{2}} .
$$

Since the virtual sound source outside the angle spanned by the loudspeakers $(\phi>\theta)$ produces $\Delta_{h}^{2}>m_{h}^{2}$, it should be noted that unlike in [4], $k_{h}^{2}$ in the closely spaced loudspeaker setup

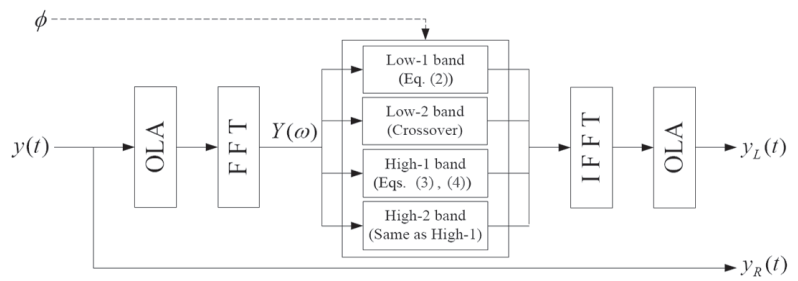

Fig. 2 Block diagram of the proposed VSI system.

can be negative because the virtual sound source outside the angle spanned by the loudspeakers produces $\Delta_{h}^{2}>m_{h}^{2}$. Thus, it is required to prevent $k_{h}$ from being an imaginary number. To this end, a real-numbered level ratio can be calculated as $k_{h}=-\sqrt{\left|k_{h}\right|^{2}}$. Or, in the frequency domain, the loudspeaker signals can be controlled as $K(\omega)=\left|k_{h}\right| e^{j\left(\omega T_{s}+\pi\right)}$. The approximated time difference $T_{s}$ can be directly applied to the pair of closely spaced loudspeakers without further modification. It should be remembered that Eqs. (3) and (4) were obtained based on the assumption that the ILDs and ITDs are constants, which, however, is not generally true for all high frequencies. Nevertheless, based on the fact that the frequency band between 3 and $4 \mathrm{kHz}$ is the most important for the high-frequency localization [10], ILDs and ITDs in that frequency band were designated as representative parameters, and applied to all high frequencies $\omega_{h} /(2 \pi)$.

Using the approximations in Eqs. (2) (4), we propose a new VSI system employing a pair of closely spaced speakers, which is depicted in Fig. 2. The approximation in the low frequency band resulted in the amplitude panning known to be effective within a frequency band up to about $600 \mathrm{~Hz}$ [11]. The high-frequency approximation model, on the other hand, is presumably applicable to the frequencies higher than $1.5 \mathrm{kHz}$. Therefore, in the proposed VSI system, the low- and high-frequency approximations are applied to the respective frequency bands where each is effective, and the approximation parameters are smoothly transited between the effective frequency bands. As a result, the frequency band is divide into four: low-1 $(0.1 \sim 0.5 \mathrm{kHz})$, low-2 $(0.5 \sim 1.5 \mathrm{kHz})$, high-1 $(1.5 \sim 5 \mathrm{kHz})$ and high-2 $(5 \sim 10$ $\mathrm{kHz})$. The band split in this paper is similar to the method in [12], but we exclude very low and high frequencies that are known to be immaterial for sound localization. Then, the parameters of low-1 and high-1 band are respectively computed using Eqs. (2), (3) and (4). The parameters of low-2 band are obtained by overlapping the low- 1 and high- 1 band parameters under the condition that the sum of the gain of each parameter is unity. Also, the parameters obtained from the $3 \mathrm{kHz}$ to $4 \mathrm{kHz}$ band are directly applied to both high-1 and high- 2 bands. Since these ILD and ITD parameters are obtained from the $3 \mathrm{kHz}$ to $4 \mathrm{kHz}$ band, splitting the highfrequency band into two is not necessary. Nevertheless, it is tried to test the relative importance of localization.

\section{Experimental Results}

The performance of the proposed VSI system was evaluated through a series of computer simulations and listening 
Table 1 Level ratios and time differences between loudspeaker signals.

\begin{tabular}{c|c|c|c}
\hline$\phi$ & $k_{l}$ & $k_{h}$ & $T_{s}(\mathrm{~ms})$ \\
\hline$-10^{\circ}$ & -3.02 & -1.95 & 0.000 \\
\hline$-20^{\circ}$ & -1.68 & -1.33 & 0.009 \\
\hline$-30^{\circ}$ & -1.42 & -1.24 & 0.015 \\
\hline$-40^{\circ}$ & -1.31 & -1.20 & 0.019 \\
\hline$-55^{\circ}$ & -1.24 & -1.18 & 0.022 \\
\hline$-65^{\circ}$ & -1.21 & -1.17 & 0.028 \\
\hline
\end{tabular}

Table 2 Average deviations from the desired IPD (radian).

\begin{tabular}{c|c|c|c|c|c|c}
\hline Low-1 band & $-10^{\circ}$ & $-20^{\circ}$ & $-30^{\circ}$ & $-40^{\circ}$ & $-55^{\circ}$ & $-65^{\circ}$ \\
\hline CTC-based & 0.01 & 0.03 & 0.05 & 0.06 & 0.07 & 0.08 \\
\hline Kirkeby's & 0.06 & 0.14 & 0.18 & 0.20 & 0.23 & 0.28 \\
\hline Proposed & 0.06 & 0.14 & 0.18 & 0.20 & 0.23 & 0.28 \\
\hline \hline Low-2 band & $-10^{\circ}$ & $-20^{\circ}$ & $-30^{\circ}$ & $-40^{\circ}$ & $-55^{\circ}$ & $-65^{\circ}$ \\
\hline CTC-based & 0.01 & 0.02 & 0.02 & 0.02 & 0.02 & 0.01 \\
\hline Kirkeby's & 0.25 & 0.47 & 0.72 & 0.78 & 0.65 & 0.57 \\
\hline Proposed & 0.12 & 0.13 & 0.18 & 0.49 & 1.13 & 1.46 \\
\hline
\end{tabular}

tests, and it was compared with those of the CTC-based [2] and Kirkeby's [6] methods. We used a stereo dipole $(\theta=$ $\left.\pm 5^{\circ}\right)$ [1] placed at a $1-\mathrm{m}$ distance $(r=1)$ from a listener. Measurements were conducted for the virtual sources localized at the angles of $\phi=-10^{\circ},-20^{\circ},-30^{\circ},-40^{\circ},-55^{\circ}$ and $-65^{\circ}$. We used the averaged HRTFs available in the CIPIC database [13]. All the systems were implemented in the frequency domain using a 2048-point FFT and the overlap-add technique. To avoid instability, the CTC-based VSI system were designed using the regularization with a parameter of 0.001 .

First, $k_{l}, k_{h}$ and $T_{s}$ parameters were computed in the stereo dipole configuration as shown in Table 1. It is noted that as the virtual source angle becomes wider, $k_{l}$ and $k_{h}$ increase to -1 , because $\tau(\omega) \gg T_{a}(\omega)$. Thus, it is anticipated that the virtual sources at wide angles may not be as accurate as those at narrow angles, or effective angles covered by the proposed system will be in a limited range.

\subsection{Evaluation of Localization Accuracy}

To objectively evaluate localization performance, we computed ILDs and ITDs of the VSI systems and compared them with the those of the ideal system. ITD was measured in term of interaural phase difference (IPD). Considering the duplex theory [7], IPDs in low-1 and low-2 bands and ILDs in high-1 and high-2 bands were investigated.

Table 2 shows the IPD deviations, calculated using $\delta_{P}=\frac{\Sigma_{\omega} \chi_{d}(\omega)-\chi_{m}(\omega) \mid \times W_{T}(\omega)}{\Sigma_{\omega} W_{T}(\omega)}$ where $\chi_{d}$ and $\chi_{m}$ are the desired and measured IPDs, respectively. To signify the perceptual aspect, the measurement was weighted by the relative transmission of sound $W_{T}(\omega)$ [14]. Results show that the CTCbased method has negligible IPD deviations. This is because the filters with enough taps can very closely invert the acoustic paths despite the regularization. Since both the proposed and Kirkeby's methods use the same low-frequency approximation, they show identical IPD deviations in low-1 band. In low-2 band, however, the proposed VSI system has smaller deviations than Kirkeby's at angles up to $\phi=-40^{\circ}$.
Table 3 Average deviations from the desired ILD (decibel).

\begin{tabular}{c|c|c|c|c|c|c}
\hline High-1 band & $-10^{\circ}$ & $-20^{\circ}$ & $-30^{\circ}$ & $-40^{\circ}$ & $-55^{\circ}$ & $-65^{\circ}$ \\
\hline CTC-based & 0.01 & 0.01 & 0.01 & 0.01 & 0.02 & 0.03 \\
\hline Kirkeby’s & 5.10 & 2.70 & 3.58 & 2.00 & 3.37 & 7.20 \\
\hline Proposed & 1.02 & 1.09 & 3.93 & 3.11 & 4.20 & 5.10 \\
\hline \hline High-2 band & $-10^{\circ}$ & $-20^{\circ}$ & $-30^{\circ}$ & $-40^{\circ}$ & $-55^{\circ}$ & $-65^{\circ}$ \\
\hline CTC-based & 0.43 & 0.20 & 0.92 & 0.92 & 1.09 & 2.93 \\
\hline Kirkeby's & 9.40 & 5.29 & 10.94 & 11.82 & 11.88 & 24.36 \\
\hline Proposed & 4.87 & 4.58 & 6.65 & 6.89 & 6.82 & 16.31 \\
\hline
\end{tabular}

Whereas, at $\phi=-55^{\circ}$ and $-65^{\circ}$, the proposed method shows larger deviations than Kirkeby's. Thus, it may be predicted that the proposed high-frequency approximation is less accurate especially beyond $\pm 55^{\circ}$, and the localization in low- 2 band is adversely influenced by the parameter smoothing. Nevertheless, as will be shown later, severe deteriorations of the localization were not perceived in the listening tests.

Table 3 summarizes the ILD deviations, computed using $\delta_{L}=\frac{\Sigma_{\omega}\left|\Delta_{d}(\omega)-\Delta_{m}(\omega)\right| \times W_{L}(\omega)}{\sum_{\omega} W_{L}(\omega)}$ where $\Delta_{d}(\omega)$ and $\Delta_{m}(\omega)$, respectively, are the desired and measured ILDs. Similarly to the IPD case, the measurements were weighted using perceptual loudness [10]. Again, the CTC-based VSI system shows very small ILD deviations. In high-1 band, ILD deviations of the proposed VSI system are smaller than Kirkeby's method at $\phi=-10^{\circ},-20^{\circ}$, similar at $\phi=-30^{\circ}$, and larger at $\phi=-40^{\circ},-55^{\circ}$. It is noted that Kirkeby's method has relatively large ILD deviations at narrow angles, because of the invalid assumption of large ILD in high frequency band. In high-2 band, the proposed system has much smaller deviations than Kirkeby's. In overall, the proposed method shows relatively regular deviations between the angles, so that it can provide more stable localization than Kirkeby's.

The localization performance was also evaluated via subjective listening tests. The tests were carried out in a $7 \mathrm{~m} \times 5 \mathrm{~m} \times 3 \mathrm{~m}$ professional listening room, which has $0.2 \mathrm{sec}$ $T_{60}$. The virtual source for the test was a 2 -second bandlimited pink noise sampled at $44.1 \mathrm{kHz}$. Twelve listeners participated in the test, and they were asked to localize virtual sources by comparing with a sound from one of five reference loudspeakers placed at $\phi=-10^{\circ},-20^{\circ},-30^{\circ},-40^{\circ}$ and $-55^{\circ}$. Since the listeners were allowed to indicate the closest reference or the center of the adjacent two references, the results were obtained at a $5^{\circ}$ resolution.

The test results are shown in Fig. 3. Regardless of the methods and the frequency bands, most listeners perceived the virtual sources to be biased toward the front, especially for wide angles, which is similar to the result in the previous study [15]. One of the main causes of this localization bias is the use of non-individualized HRTFs. Like the previous simulations, both the proposed and Kirkeby's methods showed similar performance in low-1 band, and their performances are comparable to that of the CTC-based method. In low-2 band, especially at wide angles, the proposed method performed worse than the CTC-based method. The reason can be found from the relatively large IPD deviations of the proposed method as shown in Table 2. In low-2 and high1 bands, Kirkeby's method showed slightly higher bias to 


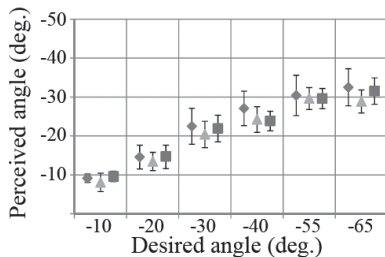

(a)

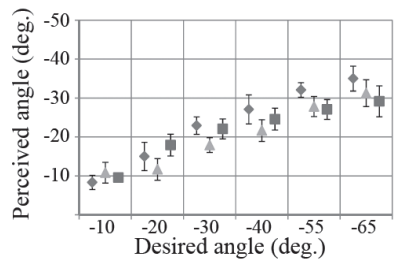

(b)

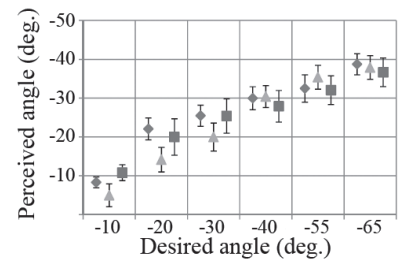

(c)

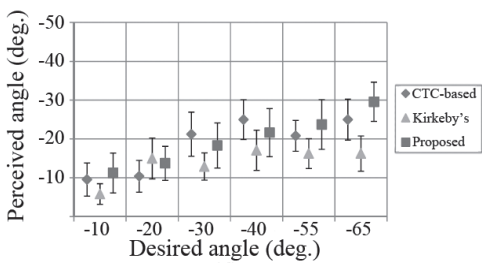

(d)

Fig. 3 Results of the listening tests: (a) low-1, (b) low-2, (c) high-1 and (d) high-2 bands.

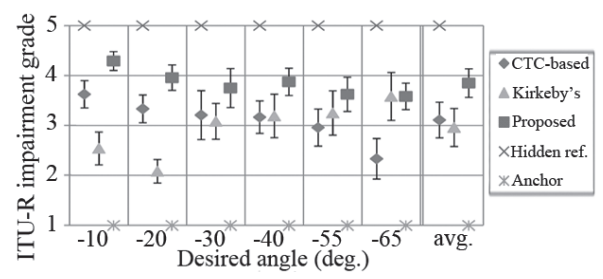

Fig. 4 Results of listening tests for sound quality.

the front than the other methods. Even with the large IPD deviations, the proposed method showed slightly better performance than Kirkeby's in low-2 band, which is partly due to the influence of the high-frequency approximation by parameter smoothing. It is also noted that the directional accuracy of the proposed method decreases as the virtual source angle increases, which is related to the parameter convergence of $k_{l}$ and $k_{h}$, as shown in Table 1. Finally, in high-2 band, most listeners found it difficult to localize the virtual sources as shown in Fig. 3(d). In summary, despite great simplification, the proposed VSI system demonstrated similar localization performance to the CTC-based VSI system in a limited range within about $\phi=-55^{\circ}$. In addition, the proposed system shows better performance than the Kirkeby's method at narrow angles up to $30^{\circ}$.

\subsection{Evaluation of Sound Quality}

Sound quality was evaluated via listening tests. The tests followed the MUSHRA-like methodology, where a 2second wide-band $(0.1 \sim 10 \mathrm{kHz})$ pink noise was used as the reference input. The anchor was generated by filtering the reference input with a $3.5 \mathrm{kHz}$ low pass filter. During the tests, subjects were asked to assess timbral variation caused by frequency distortion of the virtual source in comparison to the real source from the reference loudspeaker at each angle. The scores were expressed by ITU-R impairment grade between 5 (imperceptible) and 1 (very annoying).

Results in Fig. 4 shows that the averaged sound quality of the proposed VSI system was much higher than both Kirkeby's and the CTC-based methods. Kirkeby's method showed especially poor sound quality at narrow angles. It is mainly because of the unrealistic assumption of ILD that is inappropriate for narrow angle cases. The CTC-based method showed poor quality especially for virtual sources at wide angles. The subjective tests clearly indicate that the proposed VSI system produces much smaller timbral distortion than the other two methods.

\section{Conclusions}

In this paper, we proposed an approximated VSI system of the CTC-based method for a pair of closely spaced loudspeakers. The proposed system was expressed using representative ILDs and ITDs that were independently found in the low- and high-frequency bands. Experimental results for a stereo dipole configuration showed that, although the proposed system had a limitation of the virtual source angle, it could provide good approximations of the CTC-based method at the available angles with natural sound quality.

\section{References}

[1] P.A. Nelson, O. Kirkeby, and H. Hamada, "Sound recording and reproduction system," U.S. Patent 6, 760, 447, 1996.

[2] W.G. Gardner, 3-D Audio Using Loudspeakers, Kluwer Academic Publishers, USA, 1998.

[3] P. Minnaar and J.A. Pedersen, "Stereo widening for loudspeakers in mobile devices," AES 29th Int. Conf., Seoul, Sept. 2006.

[4] T.S. Choi, Y.C. Park, D.H. Youn, and S.P. Lee, "Virtual sound rendering in a stereophonic loudspeaker setup," IEEE Trans. Audio Speech Lang. Process., vol.19, no.7, pp.1962-1974, 2011.

[5] O. Kirkeby, "Transparent stereo widening algorithm for loudspeakers," U.S. Patent 6, 928, 168 B2, 2005.

[6] O. Kirkeby, P.A. Nelson, and H. Hamada, "Virtual source imaging using the stereo dipole," AES 103rd Convention, New York, Sept. 1997.

[7] E.A. Macpherson and J.C. Middlebrooks, "Listener weighting of cues for lateral angle: The duplex theory of sound localization revisited," J. Acoust. Soc. Am., vol.111, no.5, pp.2219-2236, May 2002.

[8] D.M. Leakey, "Some measurements on the effects of interchannel intensity and time differences in two channel sound systems," J. Acoust. Soc. Am., vol.31, no.7, pp.977-986, 1959.

[9] J. Blauert, Spatial Hearing, The Psychophysics of Human Sound Localization, revised edition, MIT Press, 1997.

[10] B.C.J. Moore, B.R. Glasberg, and T. Baer, "A model for the prediction of thresholds, loudness and partial loudness," J. Audio. Eng. Soc., vol.45, pp.224-240, 1997.

[11] V. Pulkki, "Uniform spreading of amplitude panned virtual sources," 1999 IEEE Workshop on Applications of Signal Processing to Audio and Acoustics, pp.187-190, 1999.

[12] S.L. Gay and J. Benesty, Acoustic Signal Processing for Telecommunication, Chapter 14, Kluwer Academic Publishers, 2000.

[13] V.R. Algazi, R.O. Duda, D.M. Thompson, and C. Avendano, "The CIPIC HRTF database," Proc. IEEE WASPAA, pp.99-102, New Paltz, NY, Oct. 2001.

[14] R.M. Stern and A.S. Zeiberg, "Lateralization of Complex Binaural Stimuli: A Weighted-Image Model," J. Acoust. Soc. Am., vol.84, no.1, pp.156-165, July 1988.

[15] T. Takeuchi, P.A. Nelson, O. Kirkeby, and H. Hamada, "Robustness of the performance of the stereo dipole to misalignment of head position," AES 102nd Convention, Munich, March 1997. 